\title{
Antibiotics for fever in patients without perforation after gastric endoscopic submucosal dissection and endoscopic submucosal excavation may be unnecessary: a propensity score-matching analysis
}

Yongkang Lai, Qian Zhang, Xiaolin Pan, Zhenhua Zhu, Shunhua Long, Xiaojiang Zhou, Guohua Li, Yin Zhu, Youxiang Chen and Xu Shu* (D)

\begin{abstract}
Background: Endoscopic submucosal dissection (ESD) and endoscopic submucosal excavation (ESE) have been widely used and have gradually become the main endoscopic treatment for gastrointestinal mucosal and submucosal lesions. Whether antibiotics are necessary for fever after gastric ESD and ESE remain unclear. The aim of this study was to analyse the value of using antibiotics in patients without perforation after ESD or ESE with fever.

Methods: In this retrospective study, patients with fever without perforation after ESD or ESE from January 2014 to January 2019 were included and divided into 2 groups: the antibiotic group and the non-antibiotic group. Fever and hospitalization time were compared between the 2 groups after propensity score matching.

Results: Overall, 253 patients meeting the inclusion criteria were enrolled in the present study, with 186 patients in the non-antibiotic group and 67 patients in the antibiotic group before matching, 55 patients in the non-antibiotic group and 55 patients in the antibiotic group after matching with all baseline characteristics balanced $(p>0.05)$. The duration of fever was not significantly different between the 2 groups $(p=0.12)$. However, the median hospitalization stay in the antibiotic group was longer than that in the non-antibiotic group ( 8 vs $7, p=0.007$ ).
\end{abstract}

Conclusions: Antibiotics may be unnecessary for fever in patients without perforation and without serious co-morbidities after gastric ESD or ESE.

Keywords: Endoscopic submucosal dissection, Gastric lesions, Antibiotics, Fever, Propensity score-matching analysis

\section{Background}

Endoscopic submucosal dissection (ESD) and its derived technique, endoscopic submucosal excavation (ESE), have been commonly used as minimally invasive

\footnotetext{
${ }^{*}$ Correspondence: jxmushx@126.com
}

Department of Gastroenterology, The First Affiliated Hospital

of Nanchang University, 17 Yongwaizheng, Street, Nanchang 330006,

Jiangxi Province, China treatment for gastric lesions [1-3]. Compared with conventional surgical treatment, ESD and ESE are associate with shorter operative times, shorter hospital stays and lower complication rates $[4,5]$, and long-term survival appears equivalent between ESD and gastrectomy patients [6]. However, ESD and ESE are associated with some complications, such as perforation, bleeding, and pyrexia. Among them, pyrexia is a common complication after ESD or ESE, and previous study have reported that 
fever occurs in 19.5\% of cases [7]. Bacteraemia, exposure of large wounds and long operation times may be correlated with pyrexia in patients treated after ESD or ESE [7-9]. However, the exact mechanism still needs to be investigated further.

At present, it is almost a consensus that there is no indication for prophylactic antibiotics after gastric ESD or ESE. Many studies have shown that although continuous submucosal defects caused by ESD or ESE may increase the risk of bacteraemia and/or endotoxaemia, the rate of bacteraemia after procedure remains low or the bacteraemia is transient. Therefore, the researchers concluded that prophylactic antibiotics might not be necessary for patients after ESD or ESE [8-12]. However, these studies only focused on whether antibiotics were used in advance after ESD or ESE. It remains uncertain whether antibiotics are necessary for patients with fever after gastric ESD or ESE, especially for those without perforation during or after procedure, since post-ESD bacteraemia is transient and the use of antibiotics will increase the cost of hospitalization. Relevant research is rare. Hence, the purpose of this study was to analyse whether fever after ESD or ESE should be treated with antibiotics in patients without perforation.

\section{Methods}

\section{Participants}

This is a retrospective study performed at the Department of Gastroenterology, the First Affiliated Hospital of Nanchang University in China. Patients who underwent ESD or ESE for gastric lesions at our department between January 2014 and January 2019 were enrolled. Informed consent was obtained from every patient. The exclusion criteria were as follows: (1) did not have a body temperature exceeding $37.5^{\circ} \mathrm{C}$ after ESD or ESE (regardless of the duration of the fever period); (2) age younger than 18 years or older than 85 years; (3) the use of antibiotics within 2 weeks before ESD or ESE; (4) immunodeficiency status; (5) serious cardiovascular, cerebrovascular, or hepatorenal diseases; (6) fever (temperature $>37.5{ }^{\circ} \mathrm{C}$ ) before the procedure; (7) incomplete demographic data; and (8) patients with perforation during or after the procedure.

\section{Relevant definitions}

Intraoperative bleeding refers to any bleeding in which haemoglobin is diluted from preoperative level to a level $>2 \mathrm{G} / \mathrm{dl}$ the day after ESD or ESE. Perforation is defined as other organs, extraluminal fat, or extraluminal space outside the muscle layer that can be seen through endoscopy during the ESD or ESE procedure, regardless of air accumulation in the abdominal cavity, retroperitoneum or mediastinum [13]. En bloc resection is defined as the endoscopic removal of a lesion in one piece and the acquisition of a single specimen. Procedure time is defined as the period from intraoperative marking time to withdrawal time. Fever is defined as a temperature $>37.5{ }^{\circ} \mathrm{C}$ after the procedure (regardless of the duration of the fever period). Because the fever time could not be accurately recorded in minutes, we recorded fever duration $<1$ day, $>1$ day $\leq 2$ days, $>2$ days $\leq 3$ days, $>3$ days $\leq 4$ days, and $>4$ days $\leq 5$ days as $1,2,3,4$, and 5 days, respectively.

\section{Gastric ESD and ESE procedure}

Before the ESD procedure, patients underwent an endoscopic ultrasound (EUS) test with a radial-scanning echo endoscopy unit (UM240; Olympus Co., Ltd., Tokyo, Japan) or a 12-Fr catheter probe (UM-3R, $12 \mathrm{MHz}$; Olympus Co., Ltd., Tokyo, Japan) to identify the size, shape and layer of origin of the lesion. In addition, abdominal computerized tomography (CT) was performed to evaluate the tumour location, growth pattern (intra/extraluminal) and the possibility of lateral growth or distant metastasis. All ESD and ESE procedures were performed by experienced endoscopists with more than 10 years of experience. A single-channel endoscope (GIFQ260J; Olympus Co. Ltd, Tokyo, Japan) was used in this procedure. After intravenous anaesthesia with propofol, routine vital sign monitoring was performed. After identifying the gastric lesion through endoscopy, dots were marked around them with argon plasma coagulation (APC, $40 \mathrm{~W}$ soft coagulation). Then, $250 \mathrm{ml}$ glycerol fructose, 2-3 ml indigo carmine and $1 \mathrm{ml}$ 1:10,000 epinephrine were injected into the submucosal layer to elevate the lesion. The superficial mucosa was incised along the outer edge of the marker point by endoscopists using a hook knife (KD 620LR, Olympus). Subsequently, an IT knife-2 (KD 611L, Olympus) was used to gradually separate the submucosal layer and lesion, and a snare (SD230U-20; Olympus) was used to help with the removal of the lesion if necessary. If the gastric lesions originating from submucosal layer or superficial muscularis propria (MP) layer ESE was used. ESE is the derivative technology of ESD. On the basis of ESD technology, continue to gradually peel off the submucosa and part of the muscularis propria at the base of the tumor. Hot biopsy forceps (FD-410LR; Olympus) or argon plasma coagulation (APC 300, ERBE) were used for intraoperative haemostasis. If there was active perforation caused by tumour excavation, titanium clips (HX-610-135; Aomori Olympus) or an over-the-scope clip system (OTSCs, Ovesco Endoscopy AG, Tübingen, Germany) were used to close the perforation. After removing the lesions, a stomach tube was placed based on the experience of endoscopists to 
reduce gastric pressure for at least $24 \mathrm{~h}$. All specimens were measured and immersed in formalin and were sent to the pathology department for immediate identification of the nature of the lesion.

\section{Postoperative management}

Patients were sent to our ward after recovery from anaesthesia and were asked to fast for $2-5$ days. All of the patients received infusions (electrolytes, etc.), gastric mucosal protective agents and proton pump inhibitors (PPIs). The stomach tube was removed according to each patient's condition. If patients had a fever after ESD, they were treated according to the experience of the doctors (either physical cooling, observation treatment, or use of second-generation cephalosporins for three consecutive days depending on the situation), and their temperature was tested every two hours until their temperature returned to normal. The maximum body temperature was recorded in the study. If they did not have any complications after ESD or ESE, they were permitted to return to a normal diet gradually.

\section{Statistical analysis}

We divided the patients into two groups according to whether antibiotics were used. The variables are presented as the mean \pm standard deviation (SD), the median and interquartile range (IQR) or proportion, as appropriate. Propensity score (PS) analysis was performed as a non-randomized sensitivity analysis to control and reduce the selection bias of each group. PS was estimated by using a multivariable logistic regression model with the following covariates: age, sex, diabetes, hypertension, previous abdominal surgery, lesion location, tumour size, pathology, intraoperative bleeding, operation time, en bloc resection, maximum body temperature, and stomach tube. The match ratio was $1: 1$, and the "nearest neighbour matching" method was used (calliper width $=0.1$ ). The absolute standardized difference (ASD) was used to assess the balance of covariates between the two groups. An ASD $<0.1$ signifies a good balance for a particular covariate. Then, we compared the fever days and hospitalization days between the two groups after matching.

The differences in baseline characteristics between the antibiotic and non-antibiotic groups were assessed using Student's t-test for continuous variables of a normal distribution, the chi-square test or Fisher's exact test for categorical variables, and the Wilcoxon rank-sum test for rank variables and continuous variables of an abnormally distributed, as appropriate. $p<0.05$ was considered to be statistically significant. Statistical analyses were performed using R statistical software 3.6.1 (www.r-proje ct.org) and IBM SPSS Statistics for Windows (V. 23.0).

\section{Results}

\section{Cohort characteristics}

A total of 1955 patients who had gastric lesions underwent ESD or ESD during the study period at our centre. Of these, 253 patients (12.94\%, Fig. 1) with fever and without perforation after the procedure were included in this study. Table 1 shows the baseline characteristics of the cohort. The mean age of these patients was $53.6 \pm 13.4$ years old, and $168(66.4 \%)$ patients were female. The most common pathology after gastric ESD was leiomyoma (23.7\%), followed by stromal tumor (17.4\%).

Table 2 shows the clinical characteristics of patients before and after PS. A total of 67 patients received antibiotics when they had pyrexia after ESD or ESE, while 186 did not receive antibiotics. Before PS matching, there were significant differences in 5 factors between the 2 groups, as follows: age $(p=0.014)$, hypertension $(p=0.042)$, lesion location $(p<0.001)$, maximum body temperature $(p<0.001)$ and stomach tube $(p=0.008)$. After PS matching, a total of 110 patients were paired for the analysis. There was no significant difference in the baseline characteristics between the pairs, and the scatter diagram (Fig. 2) and histogram (Fig. 3) of the tendency distribution show good matching. The ASD for all matched covariates was $<0.1$.

\section{Comparison between the non-antibiotic group and the antibiotic group}

After PS matching, the selection bias of each group was reduced and controlled. The fever durations between the two groups were compared by rank sum test, and the hospitalization time between the two groups was compared by Mann-Whitney rank sum test. The rank average of fever duration in the antibiotic group was higher than that in the non-antibiotic group, but the results were not significant (60.05 vs $48.95, p=0.12$ ). However, the median length of hospital stay in the antibiotic group was longer than that in the non-antibiotic group ( 8 vs $7, p=0.007$ ) (Table 3 ).

In the subgroup analysis, when maximum body temperature was $<38.5{ }^{\circ} \mathrm{C}$, the median hospitalization duration of the antibiotic group was still longer than that of the non-antibiotic group (8 vs $7, p=0.038$ ), and the comparison of fever duration was still nonsignificant ( 39.86 vs $49.33, p=0.33$ ) (Table 4 ). In the subgroup with a temperature $\geq 38.5{ }^{\circ} \mathrm{C}$, the comparison between the two groups in the median hospitalization duration in days ( 6.5 vs $8, p=0.14$ ) and the rank mean of fever duration in days (9.4 vs $13.25, p=0.156)$ were not significant. 


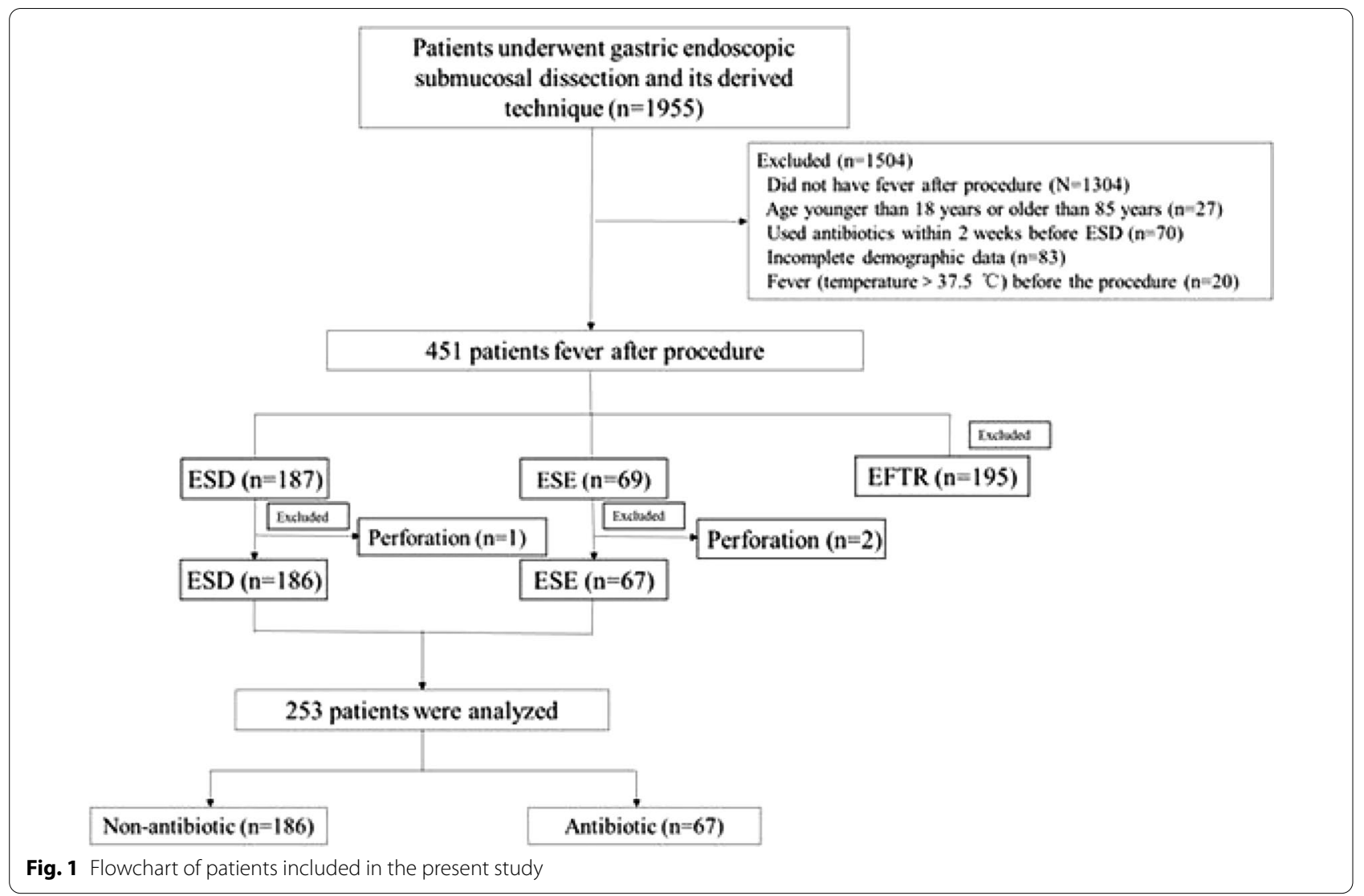

\section{Discussion}

Fever is one of the common complications after ESD and ESE, but the mechanism of fever development after ESD and ESE is still unclear. Previous studies have indicated that fever after ESD and ESE may be related to wound exposure and bacteraemia [10]. Some guidelines have recommended prophylactic use of antibiotics after ESD or ESE $[14,15]$. However, Kato et al. found that the incidence of bacteraemia after ESD was low [11]. Lee et al. found that bacteraemia after gastric ESD was temporary [9]. Other studies also concluded that prophylactic antibiotics might be unnecessary for patients with gastric ESD or ESE $[8,10]$. Since fever after ESD or ESE may be non-infectious, the necessity of antibiotics for fever after ESD or ESE is confusing, especially for those patients without perforation after procedure. The aim of this study was to analyse the need for antibiotics for postoperative fever in patients without perforation.

Before procedure, patients need complete preoperative examination, including CT, EUS, laboratory examination and so on. Hence, in our study, the median hospitalization stay is 7 (6-9) in non-antibiotic group and $8(7-10)$ antibiotic group. In the present study, the use of antibiotics did not shorten the duration of fever but increased the duration of hospitalization. This was also the case in the subgroup analysis of individuals with a temperature $<38.5{ }^{\circ} \mathrm{C}$. There was no difference in the duration of fever between the patients with a temperature above $38.5{ }^{\circ} \mathrm{C}$ who used antibiotics and those who did not use antibiotics. In addition, there was no significant difference in the number of days spent in the hospital between the two groups, which might be related to the small sample size. The mechanism of fever development after ESD and ESE is not clear. In clinical situation, some doctors will empirically use antibiotics for patients with fever after an ESD or ESE procedure. However, in this study, the use of antibiotics did not reduce the duration of fever and even increased the hospitalization stay. In addition, the use of antibiotics will also increase the cost of hospitalization and may cause adverse reactions to antibiotics, such as allergies, drug resistance and secondary infection [16-18]. We hypothesized that pyrexia after ESD or ESE is a physiological febrile response similar to that occurring after surgery [19]. The fever may be due to the release of inflammatory cytokines from macrophages, endothelial cells and the reticuloendothelial system after tissue damage, and these cytokines cause the elevation of the thermoregulatory set point for body temperature $[20$, 21]. 
Table 1 Baseline characteristics

\begin{tabular}{|c|c|}
\hline Characteristic & $N=253$ \\
\hline Age, mean $\pm S D$ & $53.6 \pm 13.4$ \\
\hline \multicolumn{2}{|l|}{ Gender [No. (\%)] } \\
\hline Female & $168(66.4)$ \\
\hline Male & $85(33.6)$ \\
\hline Diabetes [No. (\%)] & $12(4.7)$ \\
\hline Hypertension [No. (\%)] & $46(18.2)$ \\
\hline Previous abdominal surgery [No. (\%)] & $34(13.4)$ \\
\hline \multicolumn{2}{|l|}{ Lesion location [No. (\%)] } \\
\hline Upper 1/3 stomach & $70(27.7)$ \\
\hline Middle $1 / 3$ stomach & 85 (33.6) \\
\hline Lower $1 / 3$ stomach & $98(38.7)$ \\
\hline Median tumor size (mm, IQR) & $12(8-19)$ \\
\hline \multicolumn{2}{|l|}{ Pathology [No. (\%)] } \\
\hline Adenoma & $34(13.4)$ \\
\hline Adenocarcinoma & $20(7.9)$ \\
\hline Hyperplastic polyp & $24(9.5)$ \\
\hline Heterotopic pancreas & $26(10.3)$ \\
\hline Lipoma & $3(1.2)$ \\
\hline Leiomyoma & $60(23.7)$ \\
\hline Neuroendocrine tumor & $5(2)$ \\
\hline Fibroma & $7(2.8)$ \\
\hline Stromal tumor & $44(17.4)$ \\
\hline inflammation & $16(6.3)$ \\
\hline Other & $14(5.5)$ \\
\hline Intraoperative bleeding [No. (\%)] & $81(32)$ \\
\hline Median operation time(min, IQR) & $35(24-47)$ \\
\hline En bloc resection [No. (\%)] & $233(92.1)$ \\
\hline Median maximum body temperature $\left({ }^{\circ} \mathrm{C}, \mathrm{IQR}\right)$ & $37.7(37.6-38.1)$ \\
\hline$<38.5^{\circ} \mathrm{C}$ & $220(87)$ \\
\hline$\geq 38.5^{\circ} \mathrm{C}$ & $33(13)$ \\
\hline Stomach tube placement [No. (\%)] & $170(67.2)$ \\
\hline
\end{tabular}

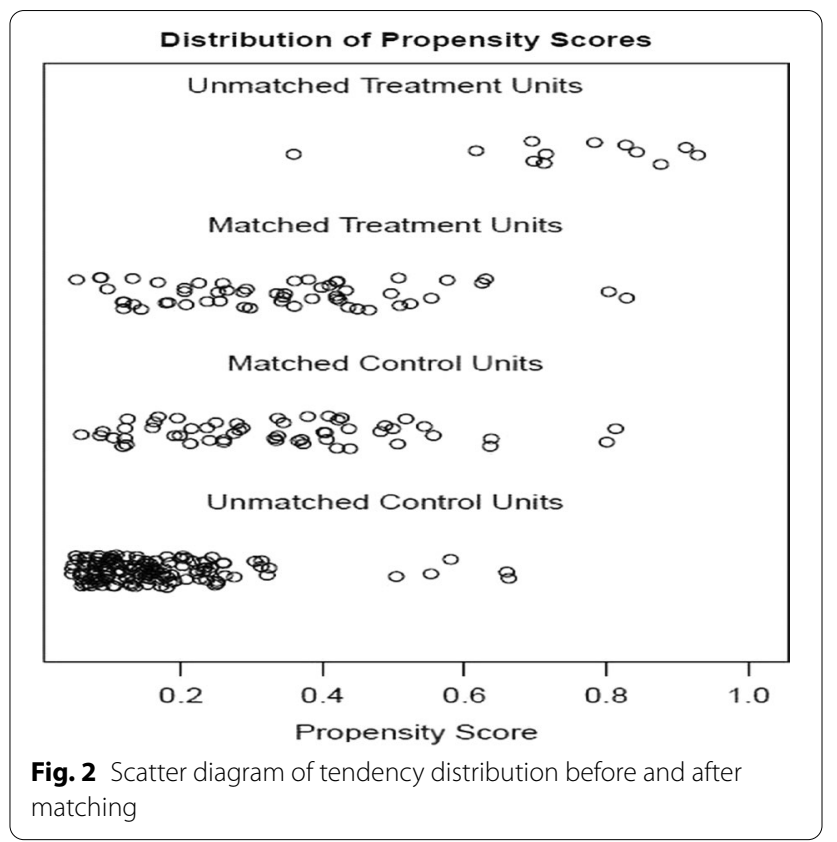

Although this was a retrospective study, our research had a large sample size, and we compared the fever time between patients in the antibiotic and non-antibiotic groups. In addition, we performed PS matching to minimize bias. Importantly, few studies have evaluated the necessity of using antibiotics for fever in patients without perforation after ESD or ESE.

There were some limitations of the present study. Firstly, the present study was a single-centre retrospective study. The findings of the present study need to be
Raw Treated

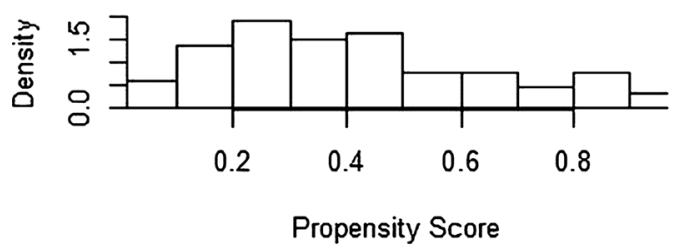

Raw Control

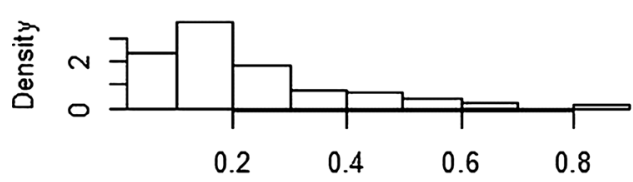

Propensity Score
Matched Treated

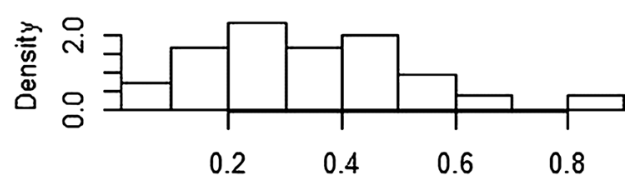

Propensity Score

Matched Control

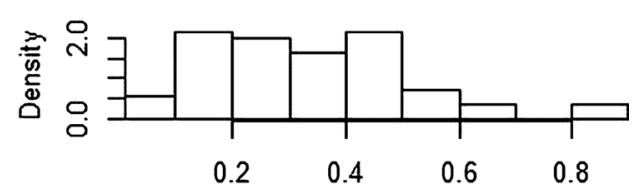

Propensity Score

Fig. 3 Histogram of tendency distribution before and after matching 
Table.2 Clinical characteristics of patients in the study before and after propensity score

\begin{tabular}{|c|c|c|c|c|c|c|c|c|}
\hline & \multicolumn{3}{|l|}{ Before matching } & \multirow[t]{2}{*}{$p$} & \multicolumn{3}{|l|}{ After matching } & \multirow[t]{2}{*}{$p$} \\
\hline & $\begin{array}{l}\text { Non- } \\
\text { antibiotics }(n=186)\end{array}$ & $\begin{array}{l}\text { Antibiotics } \\
(n=67)\end{array}$ & $\overline{\mathrm{ASD}^{\mathrm{a}}}$ & & $\begin{array}{l}\text { Non- } \\
\text { antibiotics }(n=55)\end{array}$ & $\begin{array}{l}\text { Antibiotics } \\
(n=55)\end{array}$ & $\overline{\mathrm{ASD}^{\mathrm{a}}}$ & \\
\hline Age, mean $\pm S D$ & $52.8 \pm 13.0$ & $55.6 \pm 14.5$ & 0.686 & 0.014 & $55.9 \pm 13.4$ & $54.5 \pm 14.1$ & 0.021 & 0.609 \\
\hline Gender [No. \%] & & & 0.024 & 0.763 & & & 0.022 & 0.327 \\
\hline Female & $125(67.2)$ & $143(64.2)$ & & & $31(56.4)$ & $37(67.3)$ & & \\
\hline Male & $61(32.8)$ & $24(35.8)$ & & & $24(43.6)$ & $18(32.7)$ & & \\
\hline Diabetes [No. \%] & $8(4.3)$ & $4(6)$ & 0.014 & 0.738 & $6(10.9)$ & $4(7.3)$ & 0.011 & 0.742 \\
\hline Hypertension [No. \%] & $28(15.1)$ & $18(26.9)$ & 0.022 & 0.042 & $16(29.1)$ & $14(25.5)$ & 0.02 & 0.831 \\
\hline $\begin{array}{l}\text { Previous abdominal surgery } \\
\text { [No.\%] }\end{array}$ & $24(12.9)$ & $10(14.9)$ & 0.017 & 0.835 & $7(12.7)$ & $8(14.5)$ & 0.015 & 1 \\
\hline Lesion location [No. \%] & & & 0.24 & $<0.001$ & & & 0.04 & 0.839 \\
\hline Upper $1 / 3$ stomach & $48(25.8)$ & $22(32.8)$ & & & $18(32.7)$ & $16(29.1)$ & & \\
\hline Middle $1 / 3$ stomach & $63(33.9)$ & $22(32.8)$ & & & $20(36.4)$ & $19(34.5)$ & & \\
\hline Lower $1 / 3$ stomach & $75(40.3)$ & $23(34.3)$ & & & $17(30.9)$ & $20(36.4)$ & & \\
\hline Median tumor size (mm, IQR) & $10(8-15)$ & $13(8-26)$ & 0.596 & 0.127 & $14(8-20)$ & $13(8-19)$ & 0.044 & 0.507 \\
\hline Pathology [No. \%] & & & 0.021 & 0.546 & & & 0.018 & 0.823 \\
\hline Mucosa / Submucosa & $142(76.3)$ & $51(76.1)$ & & & $41(74.5)$ & $43(78.2)$ & & \\
\hline Muscular layer & $44(23.7)$ & $16(23.9)$ & & & $14(25.5)$ & $12(21.8)$ & & \\
\hline Intraoperative bleeding [No. \%] & $54(29)$ & $27(40.3)$ & 0.024 & 0.095 & $22(40)$ & $21(38.2)$ & 0.021 & 1 \\
\hline Median operation time (min, IQR) & $33(23-43)$ & $37(24.5-56.5)$ & 0.763 & 0.076 & $37(30-51)$ & $37(21.5-50.5)$ & 0.078 & 0.183 \\
\hline En bloc resection [No. \%] & $173(93)$ & $60(89.6)$ & 0.014 & 0.428 & $54(98.2)$ & $50(90.9)$ & 0.011 & 0.206 \\
\hline $\begin{array}{l}\text { Median maximum body } \\
\text { temperature }\left({ }^{\circ} \mathrm{C}, \text { IQR }\right)\end{array}$ & $37.6(37.6-38)$ & $38(37.8-38.6)$ & 0.023 & $<0.001$ & $37.9(37.6-38.4)$ & $38(37.7-38.4)$ & 0.02 & 0.523 \\
\hline$<38.5^{\circ} \mathrm{C}$ & $174(93.5)$ & $46(68.7)$ & & & $45(81.8)$ & $43(78.2)$ & & \\
\hline$\geq 38.5^{\circ} \mathrm{C}$ & $12(6.5)$ & $21(31.3)$ & & & $10(18.2)$ & $12(21.8)$ & & \\
\hline Stomach tube placement [No. \%] & $124(66.7)$ & $46(68.7)$ & 0.024 & 0.008 & $39(70.9)$ & $38(69.1)$ & 0.023 & 1 \\
\hline
\end{tabular}

a The absolute standardized difference (ASD) was used to assess the balance of covariates between the two groups. Variables with an ASD $>0.10$ is considered to be imbalance

Table 3 Comparison between the two groups (adjustment through PS regression)

\begin{tabular}{llll}
\hline & Non-antibiotic & Antibiotic & $\boldsymbol{p}$ \\
\hline Fever time $^{\mathrm{a}}$ & $48.95^{\mathrm{b}}$ & $62.05^{\mathrm{b}}$ & 0.12 \\
Hospitalization stay $^{\mathrm{b}}$ & $7(6-9)$ & $8(7-9)$ & 0.007 \\
\hline days, median(IQR)] & & &
\end{tabular}

a Fever time was recorded as rank variables and comparison was made by Wilcoxon rank sum test

b Average rank

validated by multicentre prospective studies. Secondly, the use of antibiotics was according to the experience of the doctors, which may potentially introduce a source of bias: such as the most severe cases received antibiotics and the mild ones did not. However, this study used PS analysis to control and reduce such bias. Hence, this problem can be neglected. Thirdly, this study excluded
Table 4 Subgroup analysis of the two groups (adjustment through PS regression)

\begin{tabular}{|c|c|c|c|c|}
\hline & & Non-antibiotic & Antibiotic & $p$ \\
\hline \multirow[t]{2}{*}{$<38.5^{\circ} \mathrm{C}$} & Fever time ${ }^{a}$ & $39.86^{b}$ & $49.33^{b}$ & 0.33 \\
\hline & $\begin{array}{l}\text { Hospitalization } \\
\text { stay [days, } \\
\text { median(IQR)] }\end{array}$ & $7(6-9)$ & $8(7-10)$ & 0.038 \\
\hline \multirow[t]{2}{*}{$\geq 38.5^{\circ} \mathrm{C}$} & Fever time ${ }^{a}$ & $9.4^{b}$ & $13.25^{b}$ & 0.156 \\
\hline & $\begin{array}{l}\text { Hospitalization } \\
\text { stay }[\text { days, } \\
\text { median(IQR)] }\end{array}$ & $6.5(6-9)$ & $8(7-12)$ & 0.14 \\
\hline
\end{tabular}

a Fever time was recorded as rank variables and comparison was made by Wilcoxon rank sum test

b Average rank; hospitalization was abnormal distribution

patients with serious comorbidities, therefore, this study's results cannot be uniformly applied to all the patients and further research is needed in the future. 


\section{Conclusion}

In conclusion, doctors can choose observation treatment and antibiotics may be unnecessary for fever in patients without perforation and without serious comorbidities after ESD or ESE, for antibiotics may not have much effect on fever after ESD or ESE but will increase hospitalization duration.

\section{Abbreviations}

ESD: Endoscopic submucosal dissection; PS: Propensity score; EUS: Endoscopic ultrasound; CT: Computerized tomography; APC: Argon plasma coagulation; MP: Muscularis propria; ESE: Endoscopic submucosal excavation; OTSCs: Overthe-scope clip system; PPIs: Proton pump inhibitors; SD: Standard deviation; IQR: Interquartile range; ASD: Absolute standardized difference.

\section{Acknowledgements \\ Not applicable.}

\section{Authors' contributions}

$Y L$ collected the data, analyzed relevant information, and wrote the manuscript; QZ collected the data; XP, ZZ, SL, XZ, GL, YZ and YC clinically managed the patient. SX clinically managed the patient, designed the article and approved the final submission. All authors read and approved the final manuscript.

\section{Funding}

Not applicable.

\section{Availability of data and materials}

The datasets used and/or analysed during the current study are available from the corresponding author on reasonable request.

\section{Ethics approval and consent to participate}

The study was approved by the ethics committee of The First Affiliated Hospital of Nanchang University. Informed consent by verbal was obtained from all participants approved by the ethics committee.

\section{Consent for publication}

Not applicable.

\section{Competing interests}

All authors declare that they have no competing interests.

Received: 7 September 2020 Accepted: 4 January 2021

Published online: 12 February 2021

\section{References}

1. Goto O, Oyama T, Ono H, Takahashi A, Fujishiro M, Saito Y, et al. Endoscopic hand-suturing is feasible, safe, and might contribute in reducing bleeding risk after gastric endoscopic submucosal dissection: a multicenter pilot study (with video). GastrointestEndosc. 2020;91:1195-202.

2. Nishizawa T, Yahagi N. Long-term outcomes of using endoscopic submucosal dissection to treat early gastric cancer. Gut Liver. 2018;12(2):119-24.

3. Ahn JY, Jung HY, Choi KD, Choi JY, Kim MY, Lee JH, et al. Endoscopic and oncologic outcomes after endoscopic resection for early gastric cancer: 1370 cases of absolute and extended indications. GastrointestEndosc. 2011;74(3):485-93.
4. Pimentel-Nunes P, Dinis-Ribeiro M, Ponchon T, Repici A, Vieth M, De Ceglie A, et al. Endoscopic submucosal dissection: European Society of Gastrointestinal Endoscopy (ESGE) guideline. Endoscopy. 2015;47(9):829-54.

5. Chiu PW, Teoh AY, To KF, Wong SK, Liu SY, Lam CC, et al. Endoscopic submucosal dissection (ESD) compared with gastrectomy for treatment of early gastric neoplasia: a retrospective cohort study. SurgEndosc. 2012;26(12):3584-91.

6. Yang J, Ren M, Lu G, Liu H, Liu C, Wang W, et al. Gastrectomy versus endoscopic resection for patients with early-stage gastric adenocarcinoma: a population-based propensity matching study. J ClinGastroenterol. 2019:54:871-8.

7. Nakanishi T, Araki H, Ozawa N, Takada J, Kubota M, Imai K, et al. Risk factors for pyrexia after endoscopic submucosal dissection of gastric lesions. Endosclnt Open. 2014;2(3):E141-7.

8. Itaba S, Iboshi Y, Nakamura K, Ogino H, Sumida Y, Aso A, et al. Low-frequency of bacteremia after endoscopic submucosal dissection of the stomach. Dig Endosc. 2011;23(1):69-72.

9. Lee TH, Hsueh PR, Yeh WC, Wang HP, Wang TH, Lin JT. Low frequency of bacteremia after endoscopic mucosal resection. Gastrointest Endosc. 2000;52(2):223-5

10. Liu Y, Chen Y, Shu X, Zhu Y, Li G, Hong J, et al. Prophylactic antibiotics may be unnecessary in gastric endoscopic submucosal dissection due to the low incidence of bacteremia. SurgEndosc. 2019;34:3788-94.

11 Kato M, Kaise M, Obata T, Yonezawa J, Toyoizumi H, Yoshimura N, et al. Bacteremia and endotoxemia after endoscopic submucosal dissection for gastric neoplasia: pilot study. Gastric Cancer. 2012;15(1):15-20.

12. Li G, Zeng S, Chen Y, Zhou X, Lv N. Bacteremia after endoscopic submucosa excavation for treating the gastric muscular layer tumors. Gastroenterol Res Pract. 2015;2015:306938.

13. Fujishiro M, Yahagi N, Kakushima N, Kodashima S, Muraki Y, Ono S, et al. Successful nonsurgical management of perforation complicating endoscopic submucosal dissection of gastrointestinal epithelial neoplasms. Endoscopy. 2006;38(10):1001-6.

14 Fernandez-Esparrach G, Calderon A, De-la-Pena J, Diaz-Tasende JB, Esteban JM, Gimeno-Garcia AZ, et al. Endoscopic submucosal dissection. Sociedad Espanola de EndoscopiaDigestiva (SEED) clinical guideline. Rev EspEnferm Dig. 2014;106(2):120-32.

15. Hoteya S, Yamashita S, Kikuchi D, Nakamura M, Fujimoto A, Matsui A, et al. Endoscopic submucosal dissection for submucosal invasive gastric cancer and curability criteria. Dig Endosc. 2011;23(1):30-6

16. Munita JM, Arias CA. Mechanisms of antibiotic resistance. Microbiol Spectr 2016:4(2). https://doi.org/10.1128/microbiolspec.VMBF-0016-2015.

17. Castells M, Khan DA, Phillips EJ. Penicillin allergy. N Engl J Med. 2019;381(24):2338-51.

18 Barlam TF, Cosgrove SE, Abbo LM, MacDougall C, Schuetz AN, Septimus EJ, et al. Implementing an antibiotic stewardship program: guidelines by the Infectious Diseases Society of America and the Society for Healthcare Epidemiology of America. Clin Infect Dis. 2016;62(10):e51-77.

19. Fukuda T, Nishida M. Factors associated with physiological postoperative pyrexia. J Obstet Gynaecol Res. 2020;46(1):161-6.

20. Lin E, Calvano SE, Lowry SF. Inflammatory cytokines and cell response in surgery. Surgery. 2000;127(2):117-26.

21. Nakamura K, Matsumura K, Kaneko T, Kobayashi S, Katoh H, Negishi M. The rostral raphe pallidus nucleus mediates pyrogenic transmission from the preoptic area. J Neurosci. 2002;22(11):4600-10.

\section{Publisher's Note}

Springer Nature remains neutral with regard to jurisdictional claims in published maps and institutional affiliations. 